\title{
STUDIES ON THE PERFORMANCE EVALUATION OF PROTOCOLS IN MANETS USING SIMULATION
}

\author{
N. Thirupathi Rao ${ }^{1}$, G.Sudeepthi $^{2}$ and Debnath Bhattacharyya ${ }^{1}$ \\ ${ }^{1}$ Department of Computer Science \& Engineering \\ Vignan's Institute of Information Technology (A) \\ Visakhapatnam-530049, AP, India \\ ${ }^{2}$ Department of Computer Science \& Engineering \\ Anurag Group of Colleges, \\ Hyderabad, Telangana, India
}

nakkathiru@gmail.com, sudeepthi.govathoti@gmail.com,debnathb@gmail.com

\begin{abstract}
Mobile ad hoc networks are having no particular or fixed infrastructure for the network. It works on the IP and also works on wireless devices like machines, laptops and cell phones etc. In the process of working these networks, they don't have any mechanism for administering. These networks are very famous and will work on the basis of temporary basis. Several protocols are available for working on these sorts of networks and in the current work, we had considered the implementation of two famous protocols like DSR and RIP. In the current problem of the implementation and performance analysis, an attempt has been made to analyze the implementation and performance of the above two said both reactive and proactive routing protocols. A simulation model was developed and analyzed the performance of the current model with two above said protocols such that the use of MAC layer and also physical models. The analysis model was implemented in the network simulator ns-2 and the results and the comparison were developed in the current article.
\end{abstract}

Keywords - Reactive protocols, proactive protocols, NS-2, routing protocols, performance analysis of the network, network comparison metrics

\section{INTRODUCTION}

The versatile impromptu system is a somewhat mobile network model which is exceptionally independent and every hub has a remote handset gadget [2]. It can quickly fabricate a system in discretionary system topology structure, accomplishing continuous information transmission between hubs with no foundation [3]. It is broadly utilized in military tasks, emergency crisis correspondences, brief real occasions, conferences and different events. This announcement can be formalized by characterizing an impromptu system as a self-sufficient arrangement of versatile hosts which were interfaces by remote connections. The association of nodes frames a self-assertive correspondence chart [1,4].

In these cell systems, correspondence between two portable hubs totally depends on each other. In a MANET, no such framework exists and the system topology may progressively change in an unusual way since hubs are allowed to move [7, 8]. MANET is a self-arranging system of versatile switches associated with remote connections with no entrance point. Each cell phone in a system is independent. The cell phones are allowed to move erratically and arrange themselves subjectively. In MANET, breaking of

Received: March 25, 2019

Reviewed: May 12, 2019

Accepted: May 26, 2019

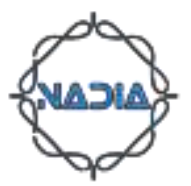


correspondence connect is exceptionally visit, as hubs are allowed to move to anyplace. The thickness of hubs and the number of hubs rely on the applications in which we are utilizing MANET $[9,10]$. MANET has offered to ascend to numerous applications like strategic systems, remote sensor position, information systems, gadget systems and so forth. With numerous applications, there are still some outline issues and difficulties to overcome.

The primary objective of portable impromptu systems administration is to broaden portability into the domain of self-sufficient, versatile, remote spaces. Where an arrangement of hubs which might be consolidated switches and they frame the system directing foundation in a specially appointed manner. A parcel of security vulnerabilities in a remote domain has been recognized and an arrangement of countermeasures was additionally proposed.

\subsection{TYPES OF MANETS}

- Vehicular appointed systems are utilized for correspondence amongst vehicles and roadside gear.

- Intelligent vehicular appointed systems are a sort of manmade brain power that encourages vehicles to act in wise behavior amid vehicle-to-vehicle crashes, mishaps.

- Smart Phone Ad Hoc Networks (SPANS) make distributed systems without depending on cell bearer systems, remote access focuses, or customary system framework.

- Ranges contrast from the customary center and talked systems, for example, Wi-Fi coordinate, in that they bolster multi-jump transfers and there is no thought of a gathering pioneer so companions can join and leave voluntarily without wrecking the system.

- Internet-based portable promotion sell systems are a sort of remote impromptu system that backings web conventions, for example, TCP/UDP and IP. The system utilizes a system layer directing convention to connect portable hubs and build up courses disseminated and consequently.

\subsection{CHARACTERISTICS OF MANETS}

In MANET, every hub goes about as both host and switch. That is it is self-governing in conduct.

1. High client thickness and Large level of client versatility.

2. Nodal availability is discontinuous.

3. Mobile specially appointed system is a gathering of independent and versatile components, for example, workstations, PDAs, wearable PCs, tablet, pc, PDA and so forth. The versatile hubs can powerfully self-arrange in discretionary brief system topology. Some primary attributes of MANET are talked about beneath.

\subsection{WIRED NETWORK PROTOCOLS}

As opposed to transporting conventions at the vehicle level (like TCP or UDP), the expression is utilized to depict a typical method to speak to data at the application level. It alludes just to a typical application layer convention and not to a typical protest semantic of the applications. Some of the wired system conventions are: 

i. Ethernet
ii. Local talk
iii. Token ring
iv. ATM

\subsection{WIRELESS NETWORK PROTOCOLS}

Wireless network, which employs elevated reappearance broadcasting influence in its place of wire to impart between hubs is one more preference for residence or trade arrange.

Some of the wireless network protocols are
i. LTE
ii. Wi-Fi
iii. Bluetooth
iv. Zigbee

Manets are to a great extent utilized as a part of the military, protect activities and even in cell phones utilizing Bluetooth. As the prominence of Manets is expanding with the expansion in logical methods, one of the greatest dangers that still dwell over Manets is security [10]. Because of its topology less nature security has turned out to be one of its primary issues. Diverse difficulties looked by Manets are [10]:
a. Privacy
b. Respectability
c. Confirmation
d. Non-renouncement lately numerous steering conventions

These protocols are divided into 3 categories.
1. Reactive
2. Proactive
3. hybrid

\subsection{PROACTIVE PROTOCOLS}

In this type of routing protocol, every hub in a system keeps up at least one directing tables which are refreshed frequently. Every hub sends a communicate message to the whole system if there is an adjustment in the system topology. It brings about extra overhead cost because of keeping up breakthrough data and subsequently throughput of the system might be influenced however it gives the real data to the accessibility of the system. The part of the aggregate control movement that comprises of genuine handy information is additionally diminished. If the hubs transmit rarely, the greater part of the steering data is viewed as repetitive. The hubs keep on expending vitality by persistently refreshing these unused sections in their directing tables as specified, vitality preservation is vital in a MANET framework outline. Along these lines, this unreasonable consumption of vitality isn't wanted. Proactive directing conventions are likewise called as table-driven steering conventions. In this, each hub keeps up steering table which contains the full data of the considerable number of hubs show in the system. The directing tables are refreshed occasionally after a little time interim.

\subsection{REACTIVE PROTOCOLS}


In this sort of coordinating tradition, each center point in a framework finds or keeps up a course in perspective of intrigue. It surges a control message by overall impart in the midst of finding a course and when the course is discovered at that point exchange speed is used for data transmission. The basic favored stance is that this tradition needs less routing information. However, the disadvantages are that it produces enormous control bundles which obviously disclosure in the midst of topology changes which happens once in a while in Manets and it realizes higher functioning. Responsive coordinating traditions were normal for these sorts of conditions [4]. These rely on the diagram that there is no point on attempting to have a photo of the entire framework topology since it will be ceaselessly developing. At whatever point a center point needs a course to a given target, it begins a course revelation process on the fly, for discovering a pathway. Responsive traditions start to set up courses on-task. The guiding tradition will try to set up such a course, at whatever point any center needs to begin correspondence with another center to which it has no course. This kind of tradition is normally greatly convincing on single-rate frameworks. It as a general rule constrains the number of hops of the picked way. Responsive directing conventions are otherwise called on-request steering conventions. These conventions don't keep up the directing data of the considerable number of hubs display in the system at constantly.

\subsection{HYBRID PROTOCOLS}

HRP is otherwise called Balanced Hybrid Routing (BHR). The crossbreed directing composition consolidates components on request and table drove steering convention. The nonspecific thought is that region where the association moderately or agreeable to table driven. It works by directing the zones with high versatility are more fitting for source started to approach. By suitably joining these two methodologies, the framework can accomplish a higher general execution.

HRP is a crossbreed convention that isolates the system into a few zones, which makes a various leveled convention [2]. HRP depends on GPS (Global Positioning System), which enables every hub to distinguish its physical position before mapping a region with table to recognize its place. The quantity of messages traded in high ZHLS is the thing that impacts the control of the transfer speed. Our convention endeavors to lessen the number of messages traded, along these lines expanding system execution and administration life. The passages diminish vitality utilization and broaden the lifetime of the bunch head in arrange.

Mixture specially appointed directing conventions this area presents a half and a half model. This model joins responsive and proactive steering conventions yet in addition, an area helped to direct convention. The zone steering convention (ZRP) [4] is a half-breed directing convention that partitions the system into zones. The intra-zone directing convention (IZRP) actualizes the steering inside the zone, while the Inter-Zone Routing Protocol (IERP) executes the steering between zones. ZRP gives a various leveled design where every hub needs to keep up extra topological data requiring additional memory. The Location-Aided Routing (LAR) [9] is an area helped steering convention that utilizations area data for the directing usefulness. LAR works correspondingly to DSR yet it utilizes area data to restrain the region where the course ask for is 14 overflowed. The beginning hub knows the neighbor's area and in view of that chooses the nearest hubs to the goal as the following jump in the course ask.

\subsection{DYNAMIC SOURCE ROUTING PROTOCOL (DSR)}

Some of the important points and features to be considered for the current protocol are as follows,

a. DSR is effective steering convention

b. It is a circle free directing convention 
c. The convention is made out of the two components of course disclosure and course upkeep, the system is totally self-sorting out and self-designing, requiring no current system foundation or organization.

d. The dynamic source directing (DSR) is one of the purest cases of an on-request steering convention that depends on the idea of source directing. It is composed particularly for use in multihop specially appointed systems of versatile hubs.

e. Discovery and course upkeep, which cooperate to enable hubs to find and keep up source courses to self-assertive goals in the system. DSR has a remarkably favorable position by the excellence of source steering. As the course is a piece of the bundle itself, steering circles, either short - lived or long - lived, can't be framed as they can be promptly identified and disposed of. This property opens up the convention to an assortment of valuable improvements.

f. It is a receptive convention and all parts of the convention work totally on-request premise. Source steering is a directing system in which the sender of a parcel decides the entire succession of hubs through which, the bundles are sent.

g. The favorable position of source steering is: moderate hubs don't have to keep up state-of-the-art directing data with a specific end goal to course the parcels they forward. DSR requires every hub to keep up a course - store of all known self - to goal sets. On the off chance that a hub has a parcel to send, it endeavors to utilize this reserve to convey the bundle.

h. If the goal does not exist in the reserve, at that point a course disclosure stage is started to find a course to goal, by sending a course ask.

i. This asks for incorporates the goal address, source address, and an exceptional recognizable proof number.

$\mathrm{j}$. If a course is accessible from the course store, however, isn't substantial anymore, a course support strategy might be started.

\section{NS-2}

NS2 is an occasion-driven recreation instrument or simulator that was helpful in concentrate the dynamic idea of correspondence systems. From that point onward, a few transformations and corrections have denoted the development of the simulator, because of significant commitments from the players in the field. Among these are the college of California and Cornell University who built up the genuine system test system, the establishment which NS depends on. Since 1995 the barrier propelled inquire about activities organization (DARPA) bolstered improvement of NS through the Virtual InterNetwork Tested (VINT) venture. Presently the national science establishment (NSF) has joined the ride being developed.

A few upheavals and amendments have denoted the development of the simulator, on account of generous commitments from the players in the field. Since 1995, the Defense Advanced Research Projects Agency (DARPA) bolstered the improvement of NS through the virtual web work tried (VINT) venture as of now the national science establishment (NSF) has joined the ride being developed. Last however not the minimum, the gathering of scientists and engineers in the group are continually attempting to keep NS2 solid and adaptable. Once more, the primary goal of this book is to give the users bits of knowledge into the NS2 design. This part gives a concise prologue to NS2. NS2 learners are prescribed to experience the nitty-gritty early on online assets. For instance, the NS2 official site gives NS2 source code and in addition point by point establishment direction.

\section{Existing System:}

Portable impromptu systems are the class of remote systems which don't require any settled foundation or base stations. Every gadget in a MANET is allowed to move freely toward any path. They can be effectively conveyed in places where it is hard to setup any 
wired foundation. The nonappearance of the settled framework in a MANET represents a few kinds of difficulties. The greatest test among them is directing. Steering is the way toward choosing ways in a system along which to send information parcels.

\section{Proposed System:}

In the current article, a simulation-based comparative analysis is performed in which RIP and DSR has been considered for investigation based on performance metrics like throughput, delay, jitter, and energy consumption using network simulator (NS2). The excellence of check is supplied to the users using NS2 for receiving straight effect examination to recognize the definite performance of the considered protocols.

\section{SIMULATION ENVIRONMENT}

The simulation experimentation is approved and carried out in LINUX environment. The comprehensive simulation model is dependent on network simulator which was used in the current scenario and its evaluation. The network simulator instructions were can be used to identify and define the topology organization of the network and the movement of the nodes in the structure, and also to organize the examiner foundation and the receiver, such that to generate the statistical data path file and so on. In the current source or the simulation environment, the continuous bit rate traffic sources are used for the current approach. The pairs that were available with the source and the destination are distributed randomly and unevenly over the network. The pairs are varied from time to time and also to be offered overall loads in the total network. The byte size used in the current network model were used in the problem are 1024 bytes of the data packets size. The mobility model used in the current article or the current problem considered was the waypoint model in the field of the rectangular model. The field which was considered in the current model of the problem was the size as $900 \mathrm{mx} 900 \mathrm{~m}$ with the combination of 140 nodes. Each packet in the current model of the network is considered as the packet starts from its journey as the random location of the source to the random destination with some random speed chosen. The simulation model was considered and the current model was executed and implemented for various seconds of 150 seconds of time. The various set of pause timings were considered for the better implementation and better utilization of the sources was done with various et of time seconds was $0,20,25,40,55,80,95,120$ seconds. The speed of the current model was implemented at the rate of speed as $14 \mathrm{~m} / \mathrm{s}$.

\subsection{AVERAGE END-END PACKET DELIVERY}

In general, the two protocols considered in the current problem RIP and DSR which can be used mostly for the available solutions to the available nodes for their utilization. The figures are shown in the current article or in the above and below sections, the commanding or the noted performance of the considered routing protocols will be analyzed and discussed for the better performance. Both the protocols are doing well in terms of performance and also the node mobility. When the nod emo0bilityis less, the convergence of the network is going or reaching up to $75 \%$ and then when there is no mobility it changes to the very high amount of convergence that is reaching up to $100 \%$.

\subsection{EFFECT OF MOBILITY}

In the existence of elevated mobility, failures of the links can occur extremely often. Failure of the links activates some new route discoveries in RIP because of its one route available for each destination or single destination. In other words, each route available for each destination in its routing table. From this, it can be understood that the number of discoveries of routes to be found on RIP was directly proportional to the breaks to be observed at the number of routes to be broken down. Whereas the observation of the DSR 
protocol for observing the failures occurring at the links is very much slow and very mild when compared with the other set of protocol. The reason that was observed in these set of the protocol was a large number of cached routes availability at the data of each node. During the performance of the DSR routing protocol, the mobility of the nodes in the network was good and high and also the cache being staling at very much stages is high when compared to another set of protocols for implementing it. Ultimately while route detection is initiated, the huge amount of replies established in reply is connected with elevated MAC slide and made augmented intrusion to information transfer.

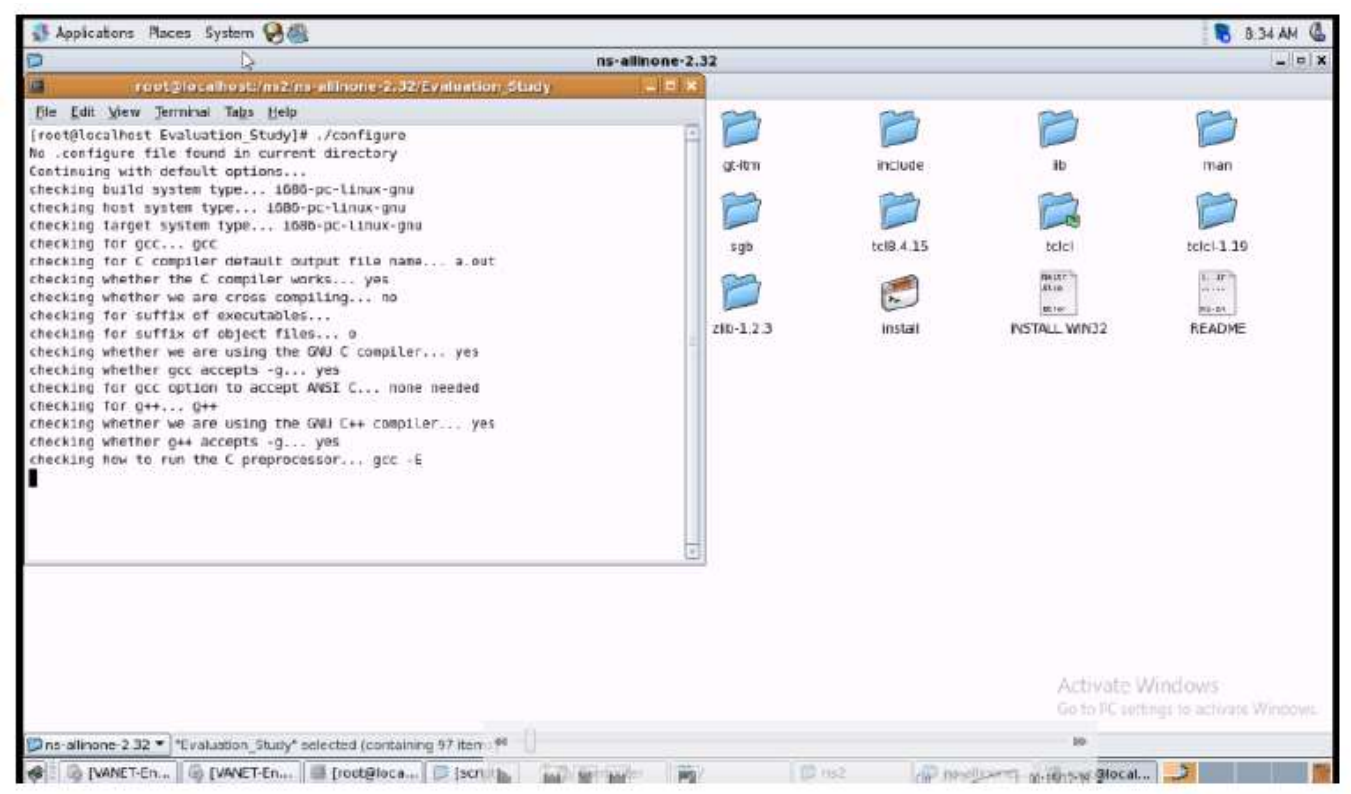

Fig. 1 Configuring the Model

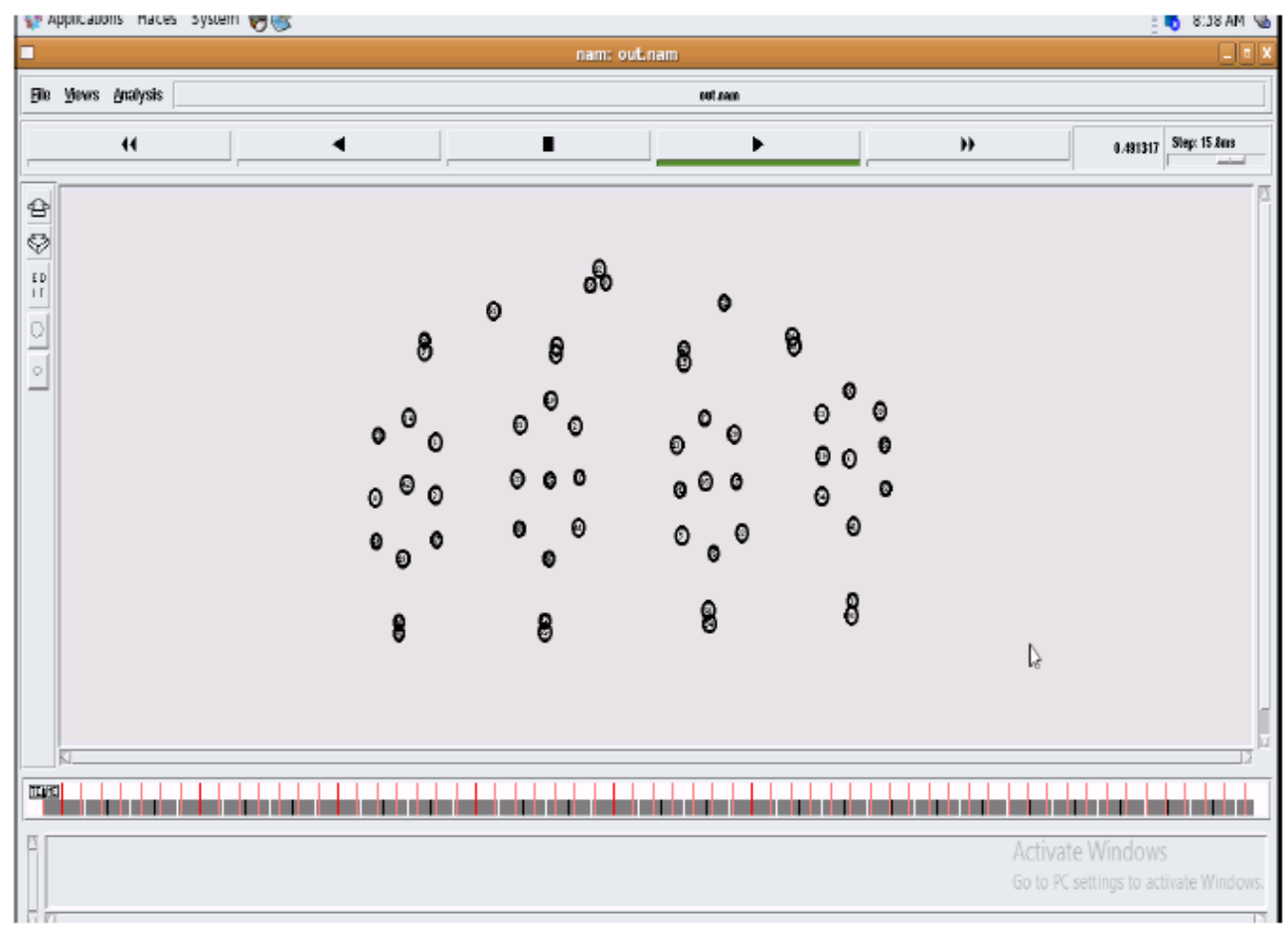

Fig. 2 Selection of nodes 


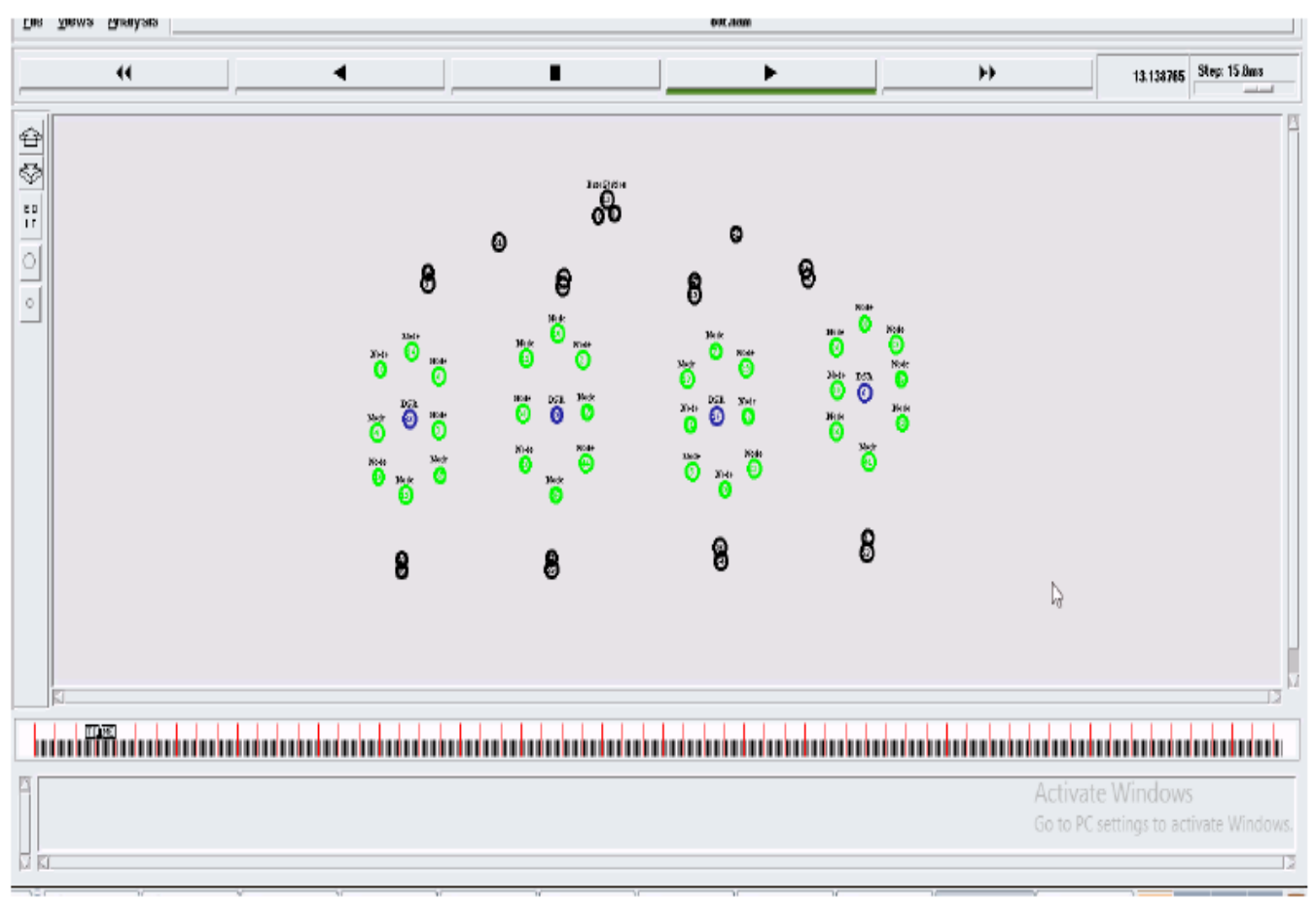

Fig. 3 Sending the packets

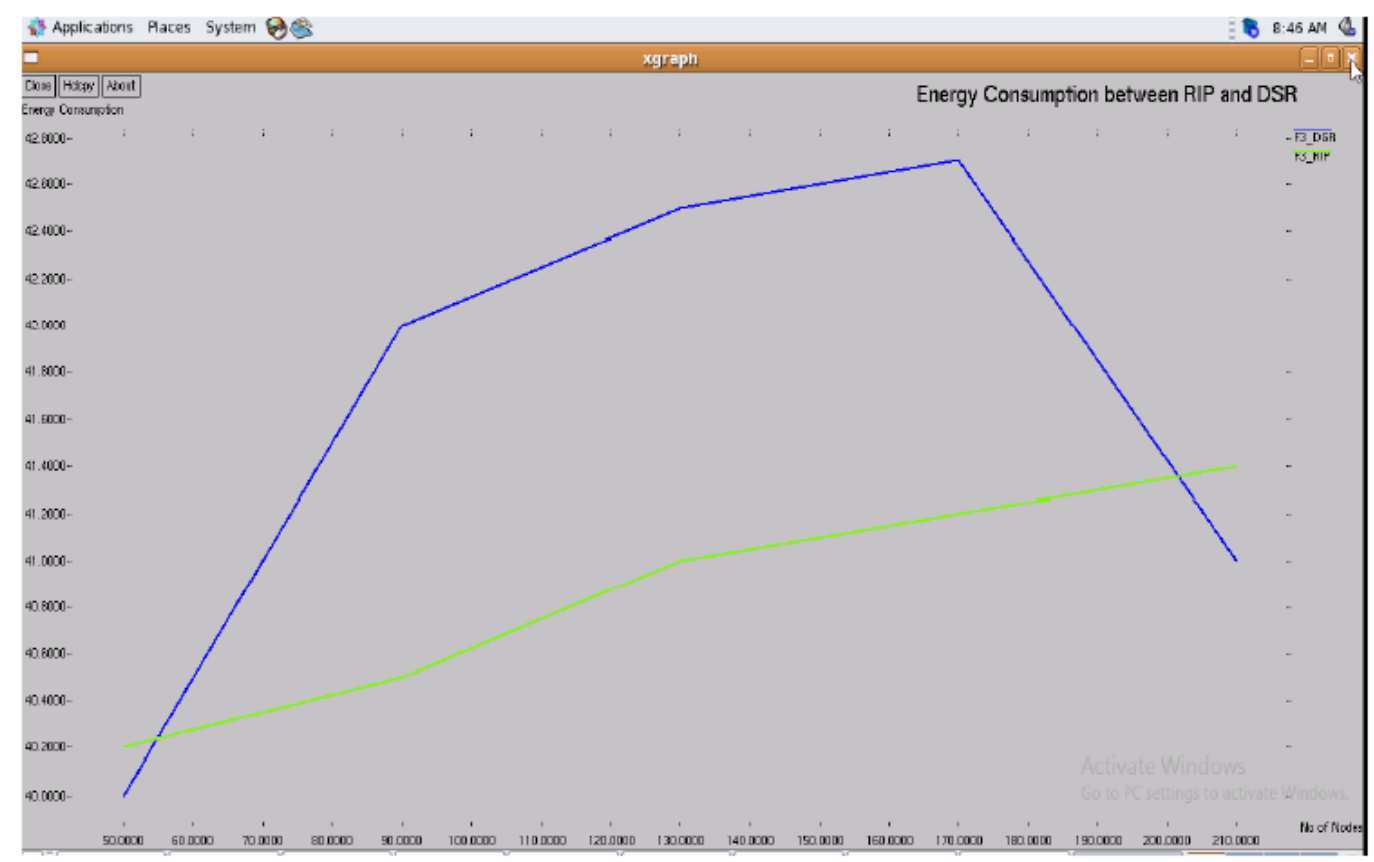

Fig. 4 Energy Consumption between both protocols 


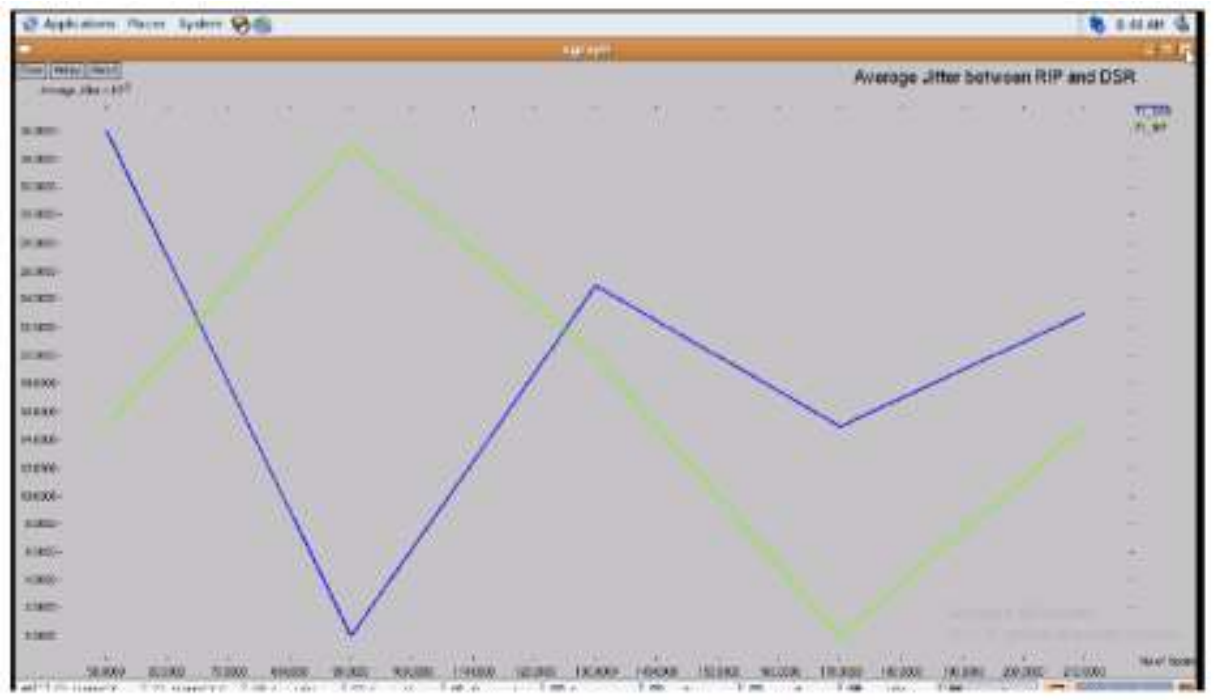

Fig. 5 Jitter between the protocols

The current work is to analyze the performance for a various set of nodes in the network in various combinations. The number of nodes considered in the network might change from time to time by considering the various set of nodes. The algorithms considered in the current article were DSR and RIP are the reactive protocols and proactive protocols. The simulation model was considered in order to study the performance of these protocols. The simulator utilized for analyzing the performance of the current considered network model was the NS-2 free simulator. These two protocols will work on the on-demand basis. Both these protocols are working very good under the condition of mobility values are at high values and the result of this mobility values high leads to the failure of the links at various times in the working of the network.

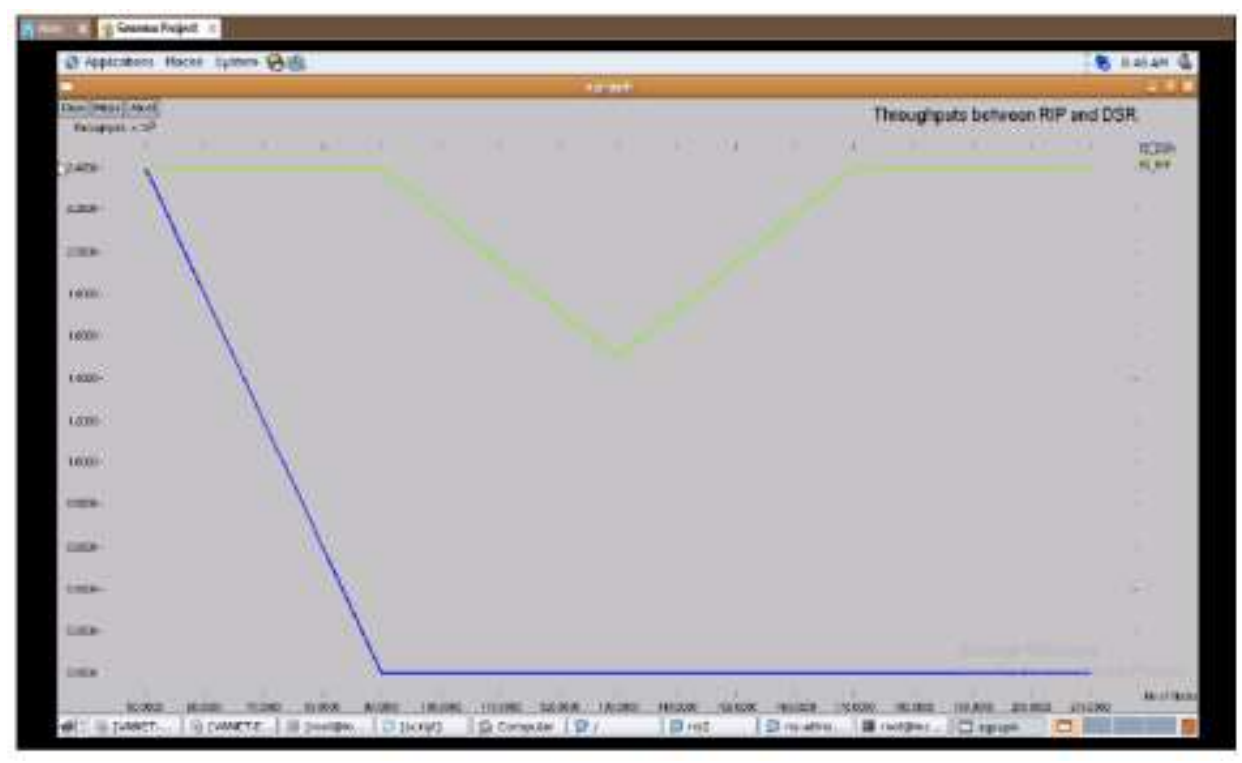

Fig. 6 Throughput between the two protocols

Also, the overhead of each routing protocol includes the updating of the new routes available and routing data available for both the protocols considered. In the case of DSR routing protocol, the current protocol uses the data of the cache and also the route cache data and this will not depend on the anytime based works or activities. On the other hand, 
the RIP protocol uses the routing table data, route destination data per one route, sequence numbers for each route destination and also it maintains the updation of the available roots. The poor performance of DSR was mostly accredited to insistent utilize of caching and be short of any method to end hard routes or decide the newness of routes when numerous options are accessible. In the current work, the performance of RIP (routing information protocol) and DSR (dynamic source routing) routing protocols in MANET.

\section{CONCLUSIONS}

The current work was considered to analyze and implement the performance of the wireless sensor network such that to study the behavior of these networks. The current work is to analyze the performance of a various set of nodes in the network in various combinations. The number of nodes considered in the network might change from time to time by considering the various set of nodes. The algorithms considered in the current article were DSR and RIP are the reactive protocols and proactive protocols. The simulation model was considered in order to study the performance of these protocols. The simulator utilized for analyzing the performance of the current considered network model was the NS-2 free simulator. These two protocols will work on the on-demand basis. Both these protocols are working very good under the condition of mobility values are at high values and the result of this mobility values high leads to the failure of the links at various times in the working of the network. Also, the overhead of each routing protocol includes the updating of the new routes available and routing data available for both the protocols considered. In the case of DSR routing protocol, the current protocol uses the data of the cache and also the route cache data and this will not depend on the anytime based works or activities.

On the other hand, the DSR protocol uses the routing table data, route destination data per one route, sequence numbers for each route destination and also it maintains the updation of the available roots. The poor performance of DSR was mostly accredited to insistent utilize of caching and be short of any method to end hard routes or decide the newness of routes when numerous options are accessible. In the current work, the performance of RIP and DSR routing protocols in MANET. In a network, it is desirable that the routing protocols should be both efficient as well as reliable. Source data send destination multi-node routing a path sending way of transmission ad-hoc network. From the results, it is observed that RIP performs better than DSR with respect to energy consumption. RIP is better than DSR with respect to end to end delay. RIP is better than DSR with respect to jitter. RIP is better than DSR with respect to throughput.

\section{REFERENCES}

[1] Md. Journeyed Islam, Et Al., "Simplified Xor Based Approach for Reducing Broadcast Redundancy with Fast Network Coverage In Wireless Ad-Hoc Networks," In $5^{\text {th }}$ International Conference on It In Asia (Cita'07), 2015.

[2] Zahra Ismail and R. Hassan, "Evaluation of Ad Hoc on Demand Distance Vector Routing Protocol In Hetman Architecture," Journal of Computer Science, Vol. 6, Pp. 830-836, 2016.

[3] Charles E. Perkins and P. Bhagwat, "Highly Dynamic Destination-Sequenced Distance- Vector Routing (DSDV) For Mobile Computers," Acm Sigcomm Computer Communication Review, Vol. 24, Pp. 234244, 2015.

[4] J Moy, "Rfc2328: OSPF Version 2," RFC Editor United States, 1998.

[5] R Colton, Et Al., "RFC 5340, OSPF for Ipv6," IETF. July, Vol. 24, 2008.

[6] G Malkin, "Rfc2453: Rip Version 2," RFC Editor United States, 2015.

[7] David B. Johnson, Et Al., "Dsr: The Dynamic Source Routing Protocol for Multi-Hop Wireless Ad Hoc Networks," Ad Hoc Networking, Vol. 5, Pp. 139-172, 2015.

[8] Charles E.Perkins, Et Al., "Ad Hoc on-Demand Distance Vector (Aodv)," Request For Comments (Rfc), Vol. 3561, 2013.

[9] Cl Hedrick, "Rfc1058: Routing Information Protocol," RFC Editor United States, 2015.

[10] G Malkin, "Ripng for Ipv6," Rfc 2080, january 2014. 